\title{
Thermal Logic Gates: Computation with phonons
}

\author{
Lei Wang ${ }^{1}$ and Baowen $\mathrm{Li}^{1,2}$ \\ 1 Department of Physics and Centre for Computational Science and Engineering, \\ National University of Singapore, Singapore 117542 \\ ${ }^{2}$ NUS Graduate School for Integrative Sciences and Engineering, Singapore 117597, Republic of Singapore
}

\begin{abstract}
Logic gates are basic digital elements for computers. We build up thermal logic gates that can perform similar operations as their electronic counterparts. The thermal logic gates are based on the nonlinear lattices, which exhibit very intriguing phenomena due to their temperature dependent power spectra. We demonstrate that phonons, the heat carriers, can be also used to carry information and processed accordingly. The possibility of nanoscale experiment is discussed.
\end{abstract}

PACS numbers: 85.90.+h 07.20.Pe 63.22.+m 89.20.Ff

As two fundamental energy transport phenomena with similar importance in nature [1], electric conduction and thermal conduction have never been treated equally. The invention of electronic transistor [2] and other relevant devices that control electric flow has led to an impressive technological development that has changed many aspects of our daily life. However, similar devices in controlling heat flow are still not available even though many experimental attempts have been made to design such devices $\underline{3},[4$, 5, [6, 7]. Recent years has witnessed some important progress, for example, thermal rectifier has been proposed by using the property of temperature dependent power spectra in nonlinear lattices [8, 9, 10, 11, 12, 13, 14, 15], and molecular level thermal machines that pump heat from a cold to a hot reservoir has been suggested 16, 17]. Merely few years after the theoretical models of thermal rectifier, a nanoscale solid state thermal rectifier has been demonstrated experimentally by asymmetrically deposited nanotubes [18]. Moreover, based on the new phenomenon:negative differential thermal resistance 9], we have also built up a thermal transistor model [19] which controls heat flow like a Field-Effect-Transistor(FET) does for the electric current.

On the other hand, phonons and other vibrational excitons in low dimensional adsorbed dielectric and semiconductor nano structures might also play an important role in information transmission [20].

In this Letter we present thermal logic gates that can do basic logic calculations. This may provide the possibility that in the near future, phonons, which are traditionally regarded as the heat carriers, can also carry information and can be processed accordingly, like electrons and photons. 'thermal computer', an alternative to the existing electronic computer, may also be possible.

In an electric/chemical 21] logic gate, power supplies that fix the voltages/chemical concentrations of some points are necessary. Similarly, in a thermal logic gate, power supplies that keep the temperatures of some points are necessary too. The temperatures of the power supplies are labeled as $T_{+}$and $T_{-}, T_{+}>T_{-}$. In any linear electric circuit in which all the resistances are con- stants, when the voltage of one node is changed by a battery connected to it, the voltage of any other node may also change, but must be in the same way, and the latter change must NOT be greater than the former one, namely, 'super response' and 'negative response' are not allowed. This is also true for thermal circuits. Therefore, nonlinear devices are necessary for any thermal logic gate.

The nonlinear device that we use in this Letter is the thermal transistor, which is depicted in Fig 1(a)). Two weakly coupled nonlinear segments, $D$ and $S$, are connected to power supplies with temperature $T_{+}(=0.2)$ and $T_{-}(=0.03)$, respectively. The control segment $G$ is coupled to the 'input signal' with temperature $T_{G}$. Each segment is modeled by a one-dimensional (1D) FrenkelKontorova (FK) lattice [22] whose Hamiltonian reads:

$$
H_{F K}=\sum_{i}\left[\frac{1}{2} \dot{x}_{i}^{2}+\frac{1}{2} k\left(x_{i}-x_{i-1}\right)^{2}+U_{i}\left(x_{i}\right)\right] .
$$

where $k$ is the spring constant and $U_{i}(x)=1-$ $\frac{V}{(2 \pi)^{2}} \cos 2 \pi x$ corresponds to the on-site potential. The FK model describes a chain of harmonic oscillators subject to an external sinusoidal potential. This is similar to the case by putting a polymer chain or a nanowire on top of adsorbed sheet[20]. Since the momentum conservation is broken in such a lattice, heat conduction obeys Fourier's law 23]. For simplicity, we have set the masses of all particles be unit. The spring constant $k$ and the on-site potential strength $V$ in different terminals are different. Terminal $D$ is coupled to terminal $\mathrm{S}$ by a spring of constant $k_{i n t}$, and terminal $S$ is coupled to control terminal $G$ by a spring of constant $k_{i n t_{G}}$. $k_{\text {int }}$ is the most important parameter of the thermal transistor. It controls the magnitude and position of the 'negative differential thermal resistance' effect discussed below. More details can be found in Fig 3(a) in ref.[19].

In our computer simulation, power supplies and input signals are simulated by Langevin heat baths and we have checked that our results do not depend on the particular heat bath realization (e.g. Nose-Hoover heat baths). We integrate the differential equations of motion by using the 
5th-order Runge-Kutta algorithm. The simulations are performed long enough to allow the system to reach a non-equilibrium stationary state and the local heat flux, $J_{i} \equiv k\left\langle\dot{x}_{i}\left(x_{i}-x_{i-1}\right)\right\rangle$, is constant in each segment. Temperature of a particle is defined as the average of twice of the kinetic energy, $T_{i}=\left\langle\dot{x}_{i}^{2}\right\rangle$.

If the lattice in segment $D$ is linear, then as temperature difference in this segment is decreased by increasing $T_{O}$, one expects an decrease in $J_{D}$. However, the FK lattice is nonlinear, one actually obtains an increased $J_{D}$ as is demonstrated in Fig 1(b). In this figure, when $T_{G}\left(T_{O}\right.$ is always very close to $\left.T_{G}\right)$ changes from $T_{o f f}(=0.03)$ to $T_{o n}(=0.14)$, the temperature difference $T_{D}-T_{O}$ is decreased, but the current $J_{D}$ increases from $2 \times 10^{-6}$ to $5.9 \times 10^{-4}$ which is about 300 times. This is the socalled negative differential thermal resistance (NDTR), an essential physical principle for thermal transistor 19]. The NDTR can be understood from studying the vibrational spectra of interface particles $O$ and $O^{\prime}$. One should recall that in our model, the heat is conducted by lattice vibration. Therefore, when one combines two segments of different lattices, the overlap of the vibration spectra of the interface particles mainly determines the heat current. If the two spectra overlap, the heat can easily go through, otherwise, it is much more difficult. The FK lattice has a temperature dependent vibrational spectrum. At the high and low temperature limits, the power spectra concentrate on low $(\omega \in[0,2 \sqrt{k}])$ and high $(\omega \in[\sqrt{V}, \sqrt{V+4 k}])$ frequencies, respectively. More details can be found in Ref. 9, 14]. As is shown in the inset of Fig.1(b), at $T_{G}=T_{o f f}$, the spectra of $O$ and $O^{\prime}$ do not overlap, thus the heat current at this point is very small, whereas at $T_{G}=T_{o n}$, the overlap is much better, thus although temperature difference at the interface is much smaller, the heat current is however much larger.

NDTR provides the keys for thermal logic gates, e.g., 'super response' and 'negative response', which are necessary for thermal signal repeater and NOT gate. See Fig 2(a), in the central region, when temperature $T_{G}$ is changed, temperature $T_{O}$ changes even greater than $T_{G}$, which makes $T_{O}$ be always closer to $T_{o n}$ or $T_{\text {off }}$ whichever is closer, than $T_{G}$ is. This can be understood by considering the direction of heat flow in segment $G$ when $T_{G}$ is slightly different from $T_{o n} / T_{o f f}$. And see Fig 2(b). $T_{O^{\prime}}$ changes in the opposite way of $T_{G}$. This can be understood by considering change of heat flow $J_{D}$ versus $T_{O}$. As $T_{G}$ increases (in this case the thermal resistance between $G$ and $O$ is very small thus $T_{O}$ is always close to $T_{G}$ ) thanks to the NDTR effect, heat flow $J_{D}$ increases, which makes temperature difference $T_{D^{-}} T_{O^{\prime}}$ increase, namely $T_{O^{\prime}}$ decreases. As have been mentioned above, these 'super response' and 'negative response' are not possible in any linear circuit. In the following, we show how to build thermal gates by combining thermal transistors. Hereafter in this paper, symbols $O, O^{\prime}, D, S$ and $G$ particularly indicate the corresponding nodes of the transistor shown in Fig:1(a).

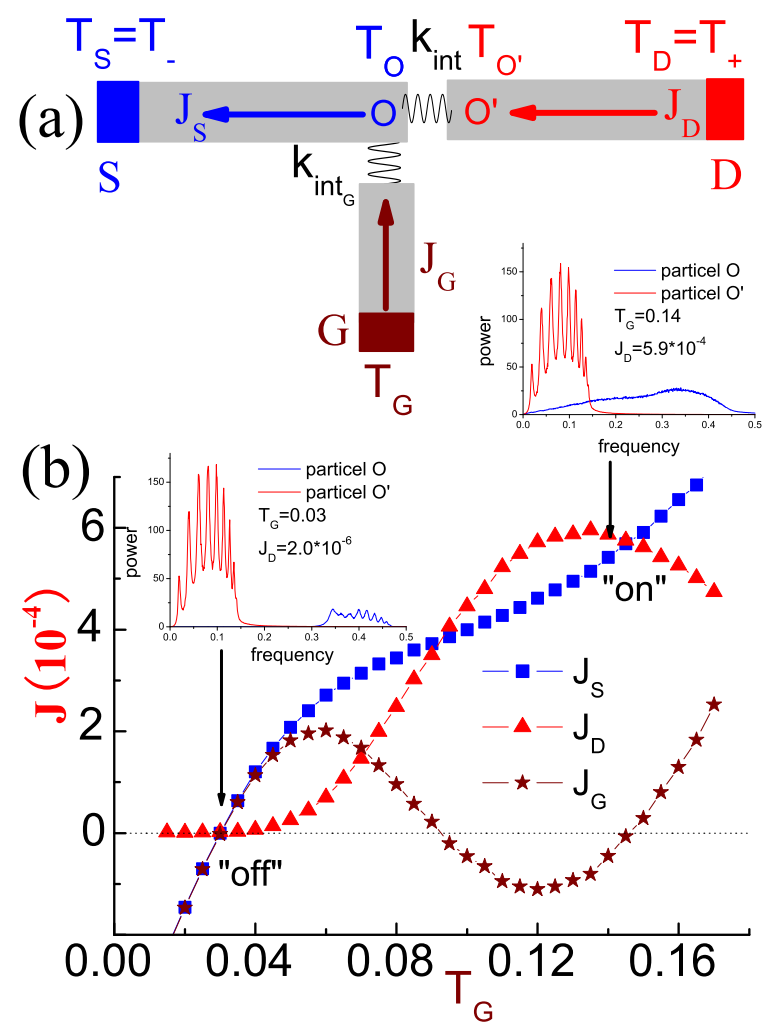

FIG. 1: (Color-online) (a) Configuration of the thermal transistor. (b) Heat currents through three terminals $D, S$ and $G$ versus temperature $T_{G}$. Notice the NDTR effect that in a wide region both $J_{S}$ and $J_{D}$ increase when temperature $T_{G}$ is increased. At $T_{G}=T_{o n}$ and $T_{o f f}, J_{G}$ is exactly zero. Insets: power spectra of particles $O$ and $O^{\prime}$ near 'off' and 'on' states. Power spectrum of $O$ depends on temperature sensitively. It matches that of $O^{\prime}$ much better at 'on' state than at 'off' state, thus makes much higher $J_{D}$ at 'on' state although temperature difference between terminal $D$ and particle $O$ is much larger at 'off' state.

In a digital electric circuit, two standard values of voltage are used to indicate states ' 1 ' and ' 0 '. Similarly we use two standard values of temperature $T_{o n}$ and $T_{o f f}$ and hereafter we fix $T_{o n}=0.16$ and $T_{o f f}=0.03$.

The most fundamental logic gate is the signal repeater, which is a two-terminal device (one input and one output) whose function is to standardize an input signal. Its response function, $T_{\text {output }}$ as a function of $T_{\text {input }}$, is:

$$
\left\{\begin{array}{l}
T_{\text {output }}=T_{\text {off }}, \quad \text { if } T_{\text {input }}<T_{c}, \\
T_{\text {output }}=T_{\text {on }}, \text { if } T_{\text {input }}>T_{c} .
\end{array}\right.
$$

Namely, when input signal is lower/higher than a critical value $T_{c}\left(T_{o f f}<T_{c}<T_{o n}\right)$ the output is exact $T_{o f f} / T_{o n}$. This is not a trivial device, without which small errors may be accumulated thus eventually leads to a wrong calculation. 


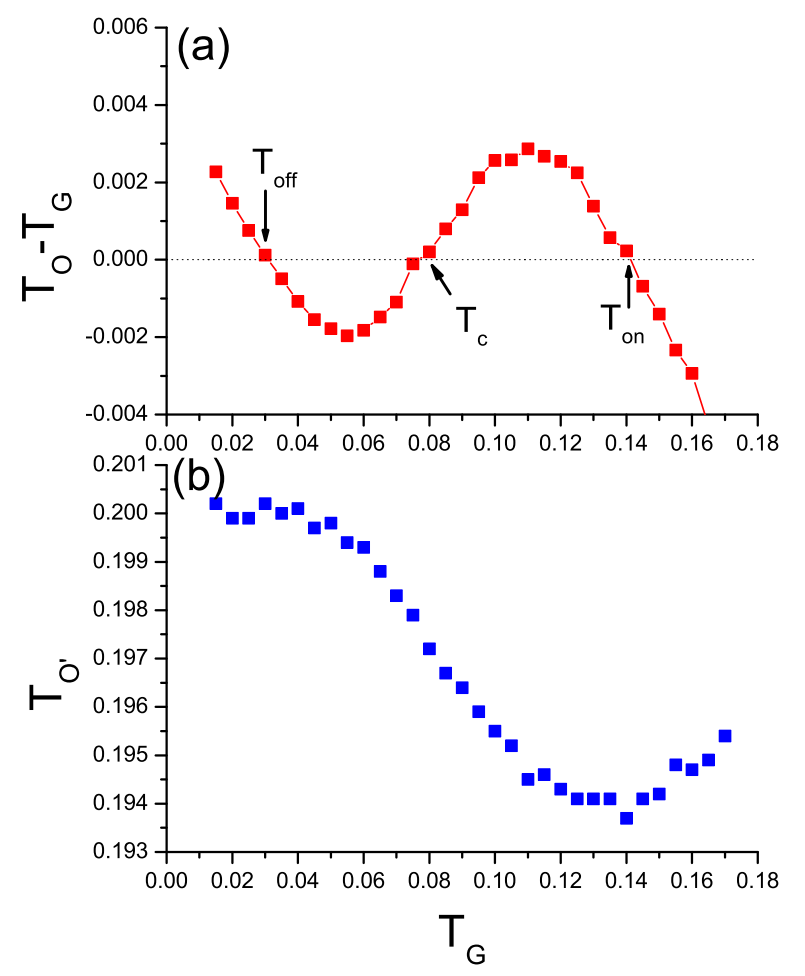

FIG. 2: (Color-online)(a) $T_{O}-T_{G}$ versus $T_{G}$. Notice the 'Super Response' that in the central region $T_{O}-T_{G}$ increases when $T_{G}$ increases, namely $T_{O}$ increases even greater than $T_{G}$ does. (b) $T_{O^{\prime}}$ versus $T_{G}$. Notice the 'Negative Response' that $T_{O^{\prime}}$ decreases as $T_{G}$ increases.

This ideal repeater can be realized by several twoterminal devices whose response functions have two stable fixed points at $T_{o n}$ and $T_{o f f}$. Therefore, if we connect them in series, namely plug the output of one device to the input of the next one, the final output is closer and closer to an ideal repeater. It is easily seen that in such a device, 'super response' i.e., $T_{\text {output }}$ changes even greater than $T_{\text {input }}$ does is necessary, otherwise the two fixed points at $T_{o n}$ and $T_{\text {off }}$ cannot be both stable. Such a device cannot be realized by any linear thermal circuit however can be simply realized by a thermal transistor by using terminal $G$ as input and node $O$ as output (Fig.2(a)). $T_{G}=T_{o n}$ and $T_{o f f}$ are the two stable fixed points and there still exists an unstable fixed point at $T_{G}=T_{c}$, which separates the attraction basins of the two stable fixed points.

As an example, we show a repeater consists of six thermal transistors in Fig 3(a). Its function is shown in Fig. 3(b). It is very close to an ideal repeater.

A NOT gate reverses the input, namely if $T_{\text {input }}=T_{\text {on }} / T_{\text {off }}$ then $T_{\text {output }}=T_{\text {off }} / T_{\text {on }}$. This requires that when $T_{\text {input }}$ increases $T_{\text {output }}$ decreases, and vice versa, which corresponds to the 'negative response', which is, as mentioned above, again realized by a thermal transistor by inputting signal to $G$ and then collecting
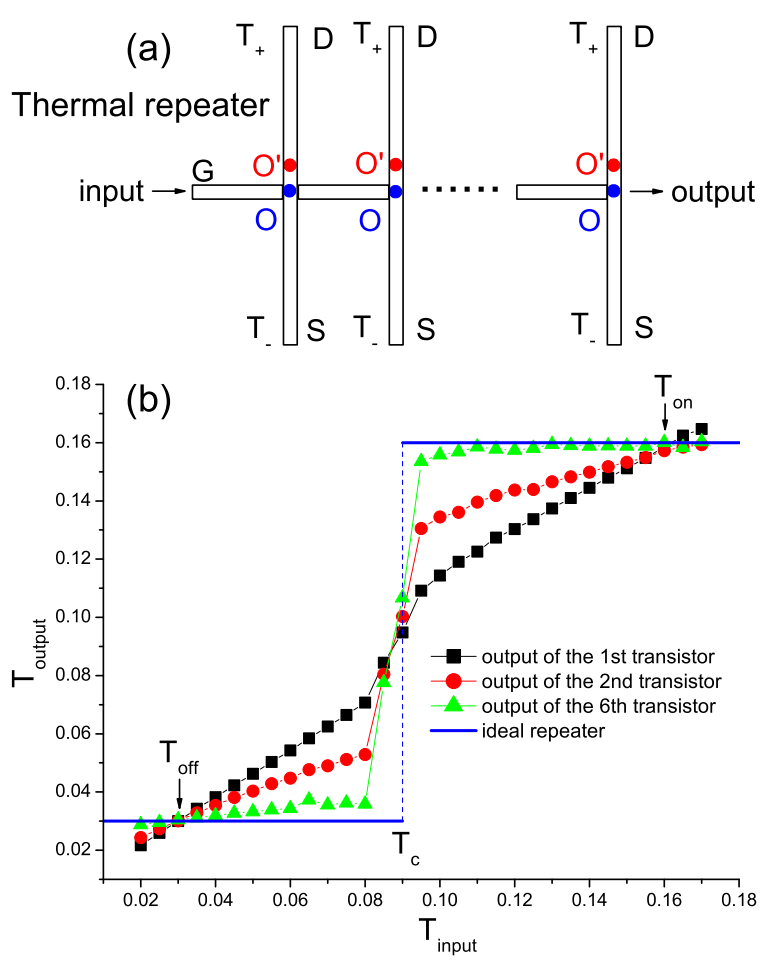

FIG. 3: (Color-online) (a) Structure of a thermal repeater. (b) Function of a thermal repeater that contains six thermal transistors. The outputs are better and better as the number of transistors is increased. The final output from the 6th transistor, is very close to that of an ideal repeater.

output from $O^{\prime}$, see Fig 2(b). Notice that $T_{O^{\prime}}$ is always higher than $T_{c}$ (in fact even higher than $T_{o n}$ ), thus will be always treated as 'on' by the next device. In order to solve this problem we use a 'temperature divider' (counterpart of a voltage divider which is shown in inset of Fig 4 (b)) whose output is a ratio of its input. By adjusting this ratio, we make its output be higher/lower than $T_{c}$ when the original input $T_{G}$ is $T_{o f f} / T_{o n}$. Then after being standardized by a thermal repeater the final output realizes the function of a NOT gate. See Fig 4 (a) and (b) for its structure and function. It is quite a surprising result because high temperature means more entropy or more randomness. Here we demonstrate that one can reduce the randomness of one part of the device by inputting more randomness in another part!

An AND/OR gate is a three-terminal (two inputs and one output) device. If one input is 'off'/'on' then the output must be 'off'/'on' regardless the other input. Otherwise if one input is 'on'/'off' then the output follows the other input. By plugging two inputs (or better after standardized by a repeater) to the same repeater, it is clear that when both the two inputs are 'on'/'off' the output must be 'on'/'off'. By simply changing some parameters of the repeaters, we can make the final output 'on'/ 'off' when the two inputs are different, therefore AND/OR 


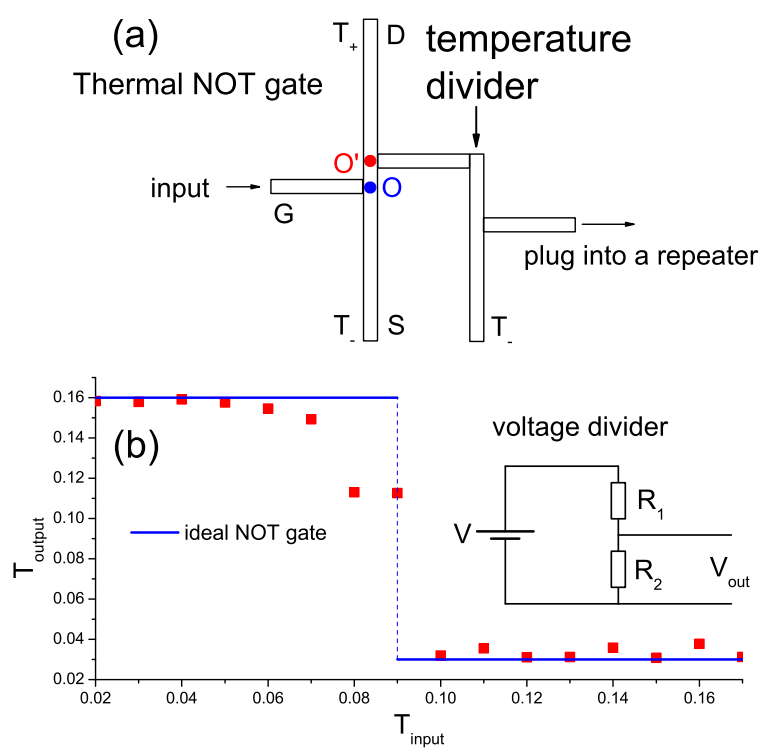

FIG. 4: (Color-online)(a) Structure of the thermal NOT gate. Through the $G$ segment, signal is transferred to $O$ point of the transistor. The output of the transistor (from $O^{\prime}$ point) is transferred to the temperature divider. Plug the output of the temperature divider into a repeater the final output is shown in (b): function of the thermal NOT gate. It is very close to an ideal NOT gate. Inset: Structure of a two-resistor voltage divider, the counterpart of a temperature divider, which supplies a voltage lower than that of the battery. Without load, the output of the voltage divider is: $V_{\text {out }}=V R_{2} /\left(R_{1}+R_{2}\right)$.

gate is realized. See Fig 5 (a) for their structures and (b) and (c) for their functions.

In summary, we have presented the feasibility to realize thermal logic calculation based on the thermal transistor model. Although the thermal logical gates given here are only toy models, they may shed light on the study of molecular information technology 24] and smart thermal materials. The study may also be helpful in understanding the complicated heat transport in biological systems.

The FK model we have used here is a very popular model in condensed matter physics and nonlinear physics [22]. It is usually used to describe energy transport in real solid. We therefore believe that our thermal logic gates can be realized in nanoscale systems experimentally in a foreseeable future, in particular, given the fact that the solid state thermal rectifier has been realized experimentally in 2006 [18], which is only a few years after the theoretical works.

The work is supported in part by an Academic Research Fund grant from Ministry of Education of Republic of Singapore.
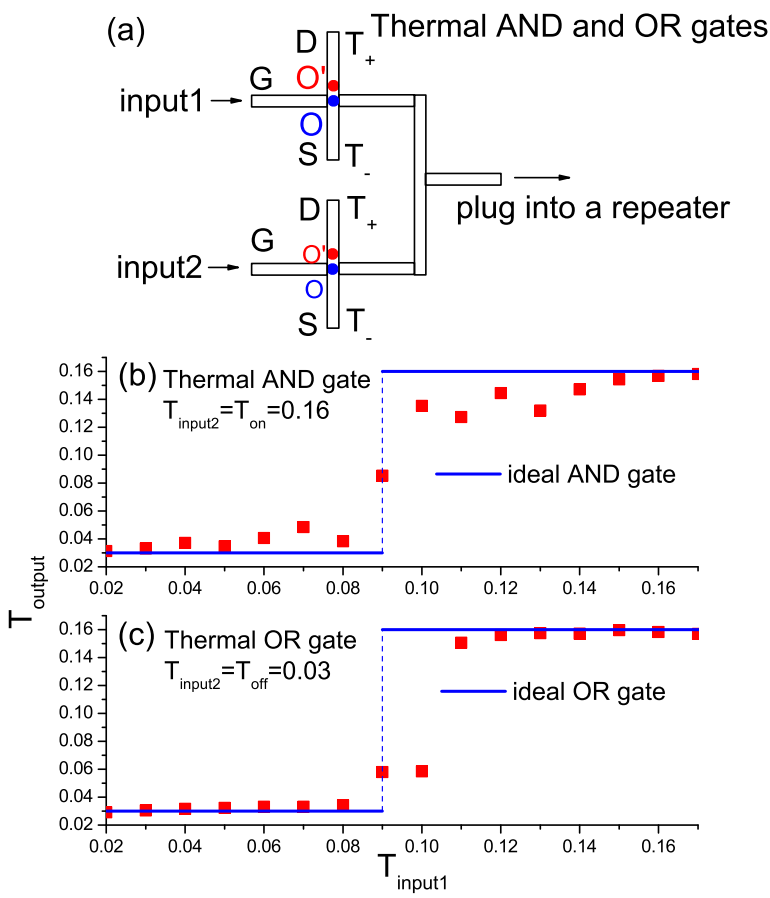

FIG. 5: (Color-online) (a) Structure of thermal AND and OR gates. Two inputs (after standardized by repeaters) are transferred to a same repeater. (b) Function of thermal AND gate. We plot $T_{\text {output }}$ versus $T_{\text {input } 2}$ while $T_{\text {input } 1}$ is fixed to $T_{\text {on }}$. (c) Function of thermal OR gate. we plot $T_{\text {output }}$ versus $T_{\text {input } 2}$ while $T_{\text {input } 1}$ is fixed to $T_{\text {off }}$.

[1] C Kittle, Introduction of Solid State Physics, 7th edition, John Wiley \& Sons, New York,

[2] J. Bardeen and W. H. Brattain, Phys. Rev. 74, 230 (1948).

[3] C. Starr, J. Appl. Phys. 7, 15 (1936).

[4] A. Williams, Ph.D Thesis, Mnchester University (1966)

[5] G. Y. Eastman, Scient. Am., 218, 38 (1968)

[6] T. R. Thomas and S. D. Probert, Int. J. Heat Mass Tranfer, 12,789 (1970)

[7] P. W. O'Callaghan, S. D. Probert and A. Jones, J. Phys. D, 31352 (1970)

[8] M. Terraneo, M. Peyrard, and G. Casati, Phys. Rev. Lett. 88, 094302 (2002).

[9] B. Li, L. Wang, and G. Casati, Phys. Rev. Lett. 93, 184301 (2004).

[10] D. Segal and A. Nitzan, Phys. Rev. Lett. 94, 034301 (2005).

[11] B. Li, J. Lan and L. Wang, Phys. Rev. Lett. 95, 104302 (2005).

[12] J. Lan and B. Li, Phys. Rev. B 74, 214305 (2006).

[13] B. Hu, L. Yang, and Y. Zhang, Phys. Rev. Lett. 97, 124302 (2006).

[14] J. Lan and B. Li, Phys. Rev. B 75, 214302 (2007).

[15] N. Yang, N. Li, L. Wang, and B. Li, Phys. Rev. 76 , 020301(R) (2007).

[16] D. Segal and A. Nitzan, Phys. Rev. E 73, 026109 (2006). 
[17] R. Marathe, A. M. Jayannavar, and A. Dhar, Phys. Rev. E 75, 030103 (2007).

[18] C. W. Chang, D. Okawa, A. Majumdar, and A. Zettl, SCIENCE, 314, 1121 (2006).

[19] B. Li, L. Wang, and G. Casati, Appl. Phys. Lett 88, 143501 (2006).

[20] V. Pouthier, J.C. Light and C. Giraredet, J. Chem. Phys.
114, 4955 (2001), and the references therein.

[21] M. O. Magnasco, Phys. Rev. Lett 78, 1190 (1997).

[22] O. M. Braun and Y. S. Kivshar, Phys. Rep. 306, 1 (1998).

[23] B. Hu, B. Li, and H. Zhao, Phys. Rev. E 57, 2992 (1998).

[24] K. P. Zauner, Critical Reviews in Solid State and materials Sciences 30 33, 2005 